\title{
Encapsulation of Rat Bone Marrow Derived Mesenchymal Stem Cells (rBMMSCs) in Collagen Type I Containing Platelet-Rich Plasma for Osteoarthritis Treatment in Rat Model
}

somayeh Ebrahimi-barough ( $\nabla$ somaye.ebrahimi@gmail.com )

Tehran University of Medical Sciences

Md Shahidul Islam

Tehran University of Medical Sciences

Mamun Al Mahtab

Bangabandhu Sheikh Mujib Medical University

Sadegh Shirian

Shahrekord University

Hamid Reza Aghayan

Tehran University of Medical Sciences

Babak Arjmand

Tehran University of Medical Sciences

Amir Allahverdi

Tehran University of Medical Sciences

Faezeh Esmaeili Ranjbar

Tehran University of Medical Sciences

Amin Bigham Sadegh

Shahrekord University

Jafar Ai

Tehran University of Medical Sciences

\section{Research Article}

Keywords: Osteoarthritis, Tissue engineering, Mesenchymal stem cells, PRP, Collagen type I

Posted Date: September 16th, 2021

DOl: https://doi.org/10.21203/rs.3.rs-875172/v1

License: (c) (1) This work is licensed under a Creative Commons Attribution 4.0 International License. Read Full License 
Page 2/24 


\section{Abstract}

Osteoarthritis (OA) is the most common form of degenerative joint disease, affecting more than $25 \%$ of the adult though prevalent in the elderly population. Most of the current therapeutic modalities aim at symptomatic treatment and lingering the disease progression. In recent years, regenerative medicine such as stem cell transplantation and tissue engineering has been suggested as a potential curative intervention for $\mathrm{OA}$. The objective of current study was to assess the safety and efficacy of an injectable tissue-engineered construct composed of BMMSCs, PRP, and Collagen type I in rat model of OA.To produce collagen type I, PRP and BMMSCs, male Wistar rats were ethically euthanized. After expansion and characterization of rat BMMSCs (rBMMSCs), tissue-engineered construct was formed by combination of appropriate amount of collagen type I, PRP and rBMMSCs. In vitro studies were conducted to evaluate the effect of PRP on chondrogenic differentiation capacity of encapsulated cells. Then tissue-engineered construct was injected in knee joint of rat model of OA (24 rat in 4 groups:OA, $\mathrm{OA}+\mathrm{MSC}, \mathrm{OA}+\mathrm{Collagen+MSC+PRP,OA+MSC+Collagen).After} 6$ weeks, the animals were euthanized and knee joint histopathology examinations were performed to evaluate the effect of each treatment on OA.Tissue-engineered construct was successfully manufactured and in vitro assays demonstrated that relevant chondrogenic genes and proteins expression were higher in PRP group than the others. Histopathological findings of the knee joint samples showed favorable regenerative effect of rBMMSCs+PRP+Collagengroup comparing to others.In this study, we introduced an injectable tissueengineered product composed of rBMMSCs+PRP+Collagenwith potential regenerative effect on cartilage damage caused by $O A$.

\section{Introduction}

In the last decades, advancement in health care system improved the prevention, diagnosis and treatment of great number of disorders which restrict human life expectancy [1,2]. These advancements led to population aging and high prevalence of degenerative disorders same as osteoarthritis (OA). OA considered as the fourth leading cause of physical disability in both the young and the old people in the world. Conventional medical treatment approaches for $\mathrm{OA}$ are focused mainly on pain reliefand symptom management including nonsteroidal anti-inflammatory drugs (NSAIDs), physical exercises and braces. Besides, some invasive curative strategies consist of steroids, hyaluronic acids (HA), platelet-rich plasma (PRP) injection. When Conventional treatments strategies fail, final curative option may be the surgical approaches [3]. Unfortunately, there are no effective therapeutics, hence,scientistsattempt to find novel treatment to restore the function and structure of damaged articular cartilage. Regenerative medicine has been exploiting tissue engineering approaches to treat OA for decades. Numerous pre-clinical and clinical studies have been conducted using SVF, PRP and Stem cells of various kinds (especially mesenchymal in origin) either single or in combination [1]. A few studies revealed that the transplanted stem cells die within a few days after transplantation due to a harsh environmental conditions (e.g., nutrient and oxygen deprivation, oxidative stress, mechanical stress) coupled with death signals, inflammation and anoikis, a programmed cell death due to loss of anchorage to the ECM. [2, 3]. Tissue engineering is an attractive 
field to regenerate damaged tissues and restore their functions with three components including cells, 3D scaffold and growth factors. Recently, the autologous chondrocyte implantation (ACl) has appeared as a promising cell-based therapy aim to provide functional repair of injured cartilage to manage the disease symptoms [4]. In fact,AClapproach is restricted by some hindrances including insufficient donor tissue supply, donor site injury and the rapid dedifferentiation of transplanted chondrocytes during twodimensional expansion [5]. To overcome such obstacles, tissue engineering recommends scaffolds in combination with stem cells as poly-therapy to provide three dimensional environment[6].Multipotent mesenchymal stem cells (MSCs) with chondrocyte differentiation capacity proposed as an attractive source to OA injuries regeneration (direct intra-articular injection) [7-9]. Also, the homing ability of MSCs to injured sites has been shown [10] and after transplantation exerted its effectiveness to cartilage repair $[11,12]$. One limitation of utilizing MSCsalone is insufficient ratio of implanted MSCs and chondrogenic differentiation difficulty.Leijset al demonstrated the low homing of injected MSCs into cartilage while the majority of MSCs detected in joint space or adhered to synovial membrane [13]. Unlike MSCs transplantation, tissue engineering strategies using stem cells seeded or encapsulated in appropriate scaffold such as hydrogels which is impregnated with growth factors revealed more acceptable results for cartilage repair $[10,14,15]$. In cartilage tissue, chondrocytes are encapsulated in a thin rim named pericellular matrix (PCM) that play a critical role in chondrocyte anchorage to the extracellular matrix and preserving chondrogenic differentiation capability $[16,17]$. The PCM and the entrapped chondrocyte make functional cartilage unit termed 'chondron'. However, the injected stem cells are encapsulated with a biomimetic scaffold can facilitate cell migration to a targeted niche and promote chondrogenic potential capability [17]. For clinical application, the cells should be encapsulated in an injectable biocompatible scaffold with low immunogenic reactionwhich could beeasily prepared in large scale.Biomaterials like hydrogel mimic ECM that provides support to vulnerable stem cells through cell-tocell adhesion, proliferation and adherence to host cells. Collagen, one of the different hydrogels is a major component of ECM. Study shows that collagen successfully improved the MSCs viability and enhanced chondrogenic differentiation [4]. Our study precisely opted bone marrow derived mesenchymal stem cells encapsulated with collagen type I in combination with PRP in order to reduce inflammation, promote chondrogenic differentiation to repair OA in rat model successfully. After extensive literature review, we found this combinatorial approach is quite unique, novel and scientifically sound.Because of the great importance of this factor, collagen type I appears to be a hopeful candidate with PCM mimicry to form an injectable chondron. MSCs encapsulated in collagen hydrogel reside in the specific niche and differentiate to chondrocyte properly $[18,19]$. Incorporation of collagen hydrogel in various tissueengineered-based scaffolds has gained priority in recent studies in order to increase the biocompatibility, biodegradability, and regenerative potential [6].Collagen type I has a high connection affinity to fibronectin (FN) that is expressed at the damaged sites so collagen hydrogel provide a biocompatible injectable scaffold to find cartilage damaged and aim to chondrocyte differentiation promotion of encapsulated MSCs [20, 21].Besides, the injected cells need adequate growth factors to accelerate their differentiation and conduct them to their determined fate. Recent studies detected the regenerative therapeutic effects of PRP because of the great number of growth factors presence that increase healing rate of damagedtissues by growth promotion in the site of injection [22-24]. In this study, we preparedan 
injectable collagen hydrogel that contains MSCs and PRP to evaluate the effect of this tissue-engineered construct transplantation in rat model of osteoarthritis.

\section{Materials And Methods}

\section{Experimental animals}

MaleWistar rats (2 months old, 200-220g) were purchased from the animal house of Pasteur Institute (Tehran, Iran). The study was performed according to instructions approved by the Animal Care and Use Committees of Tehran University of Medical Sciences (ethical code: IR.TUMS.

VCR.1398.034).Theanimals were kept in a room with controlled temperature $\left(21-25^{\circ} \mathrm{C}\right)$ on a 12 hours light/12 hours dark cycle.

\section{Rat bone marrow mesenchymal stem cells (rBMMSCs) isolation and culture}

Adult rats were euthanized under proper anesthesia with ethically approved protocol. Femora and tibias were harvested aseptically. Connective tissues were removed and both ends of each bone were cut. Bone shafts were thoroughly washed with sterile PBS and the bone marrow were flushed-out with Dulbecco's Modified Eagle's Medium (DMEM). The cell suspension was collected in a $90 \mathrm{~mm}$ culture dish, then filtered by strainer $(70 \mu \mathrm{m})$ and centrifuged at $1200 \mathrm{rpm}$ for 5 minand re-suspended in growth medium containing low glucose DMEM supplemented with $10 \%$ FBS, penicillin $(100 \mathrm{U} / \mathrm{mL})$, L-glutamine $(2 \mathrm{mM})$, streptomycin $(100 \mu \mathrm{g} / \mathrm{mL})$, and amphotericin-B $(0.25 \mu \mathrm{g} / \mathrm{mL})$ (all from Thermo Fisher Scientific, USA). Finally, cells were plated at a density of $10^{5} \mathrm{cells} / \mathrm{cm}^{2}$ in a T25tissue culture flask (Nunc, USA) and maintained at $37^{\circ} \mathrm{C}$ in a humidified atmosphere of $5 \% \mathrm{CO}_{2}$. The medium was refreshed after 24 hours and then every 3 days. Cells at $70-80 \%$ confluency were serially passaged. We used cells at passage 3 for subsequent experiments.

\section{rBMMSCs characterization}

To characterize the cell population as multipotent MSCs, Cells at $3^{\text {rd }}$ passage were characterized to meet the minimal criteria such as morphology, plastic-adherent property, expression of MSC-specific surface markers by flow cytometry and the ability for multi-lineage differentiation in vitro.

\section{Surface marker expression}

Flow cytometery(FACS Calibur, BD Bioscience, USA) was performed to determine the phenotypic expression of rBMMSCs. At 3rd passages, $1 \times 10^{6}$ cells were stained with anti-CD-90, anti-CD-105, anti-CD45, and anti-CD-31 (All from Abcam, USA) We used the following antibodies: primary antibodiesanti-CD105 mouse monoclonal (ab2529), anti-CD-90 mouse monoclonal (ab222781), anti-CD-34 mouse monoclonal (ab81289), and FITC-conjugated anti-CD-31 mouse monoclonal (ab33858). Unstained cells were used for iso type control. Data wereanalyzed by recording 10,000 events using FlowJo Version 7 software. 


\section{In vitro differentiation of rBMMSCs}

After three passages, cells were re-plated in growth medium at $2 \times 10^{5}$ cells/well in 24 -well tissue culture plates. After $24 \mathrm{~h}$ incubation, the growth medium was replaced with osteogenic differentiation medium containing dexamethasone (10nmol), $\beta$-glycerophosphate $(10 \mathrm{mmol})$, L-ascorbic acid $(0.3 \mathrm{mM})$ (all from

Sigma-Aldrich, USA). For adipogenic differentiation, $2 \times 10^{5} \mathrm{rBMMSCs} /$ well were cultured in 24-well tissue culture plates and after $24 \mathrm{~h}$ incubation, adipogenic differentiation media containing insulin $(10 \mu \mathrm{L} / \mathrm{mL})$, dexamethasone $(1 \mu \mathrm{M})$, indomethacin $(0.5 \mathrm{mM})$, and 3-isobutyl-1-methylxanine $(60 \mu \mathrm{M})$ (all from SigmaAldrich, USA) was added.The humidified atmosphere with $5 \% \mathrm{CO}_{2}$ was maintained all through the incubation. The differentiation media was replaced every three days. After 21 days of differentiation period, the media was removed and cells were fixed and stained by alizarin red for osteoblast cells and Oil Red $\mathrm{O}$ staining for adipocyte cells. Calcium deposition and lipid droplets in the cells were observed using microscopy.

\section{PRP preparation and activation}

Approximately $8 \mathrm{ml}$ blood was collected from each euthanized rat by cardiac puncture and transferred to a centrifuge tube pre-loaded with $2 \mathrm{ml}$ acid citrate dextrose solution. The tubes were then centrifuged at $2000 \mathrm{rpm}$ for $10 \mathrm{~min}$ at $20^{\circ} \mathrm{C}$ and plasma collected carefully then further centrifuged at $4000 \mathrm{rpm}$ for 10 min at same temperature. The supernatant alone is platelet-poor-plasma (PPP) and the precipitate at the bottom of the centrifuge tube with supernatant is the PRP. When PRP volume was more than $2 \mathrm{~mL}$, amount $1 \mathrm{ml}$ of PRP was used for blood count. We activated PRP immediate prior to application with $\mathrm{CaCl}_{2} 10 \%\left(0.2 \mathrm{ml} \mathrm{CaCl}_{2}\right.$ to $\left.0.8 \mathrm{ml} \mathrm{PRP}\right)$.

\section{Collagen type I gel preparation and cell encapsulation}

Collagen type I was extracted following the protocol described by NavneetaRajan and colleagues (25)from rat tails and processed using acetone, $70 \%$ (vol/vol) isopropanol, and $0.02 \mathrm{~N}$ cold acetic acid. The pure acetone, isopropyl alcohol, acetic acid were purchased from Merck (Germany).The collagen solution was sterilized using $1 \%$ chloroform(Merck (Germany)) and the resultant solution was mixed with 10X DMEM-F12(Gibco, USA) and triple-buffer system-HSS at a volume ratio of 8:1:1 vol/volrespectively. The medium and HSS buffer were filtered through $0.22 \mu \mathrm{m}$ strainer prior to mixing. The HSS buffer composed of HEPES $4.77 \mathrm{~g}$ and $2.2 \mathrm{~g}$ sodium bicarbonate in $100 \mathrm{~mL}$ of $0.5 \mathrm{M} \mathrm{NaOH}$ solution.All the components of HSS were purchased from Sigma Aldrich (USA). The final concentration of collagen type I hydrogel was $2 \mathrm{mg} / \mathrm{ml}$.

rBMMSCs encapsulation procedure is shown in graphical abstract. Briefly, the activated PRP and collagen type I solution were mixed properly $(1: 1 \mathrm{vol} / \mathrm{vol})$ in a falcontube and kept half an hour in room temperature. MSCs were harvested by using trypsin/EDTA and $2 \times 10^{6}$ cellssuspended in $100 \mu \mathrm{L}$ of complete culture media. The cell suspension was added to PRP-Collagen solution andmixed by gentlepipetting. The composition transferred and cultured in 24-well plate. 


\section{Cell morphology and attachment by SEM}

The attachment of rBMMSCs in collagen was investgated by scanning electron microscope (SEM) after $48 \mathrm{~h}$ post cell seeding. For SEM analysis the samples were were fixed by an aqueous solution of Karnovsky's fixative (consisting of $2.5 \%$ glutaraldehyde and $2 \%$ paraformaldehyde) for 90 min at room temperature. Thereafter, the prepared specimens were washed two times with PBS and dehydrated using a graded ethanol series of $30,50,70,90,95$, and $100 \%$. Finally, the specimens were dried under vacuum and after coating with gold, examined via SEM (SEM, AIS2300C SEI, Korea) at $20 \mathrm{kV}$.

\section{MTT assay}

MTT assay was performed to measure the viability of encapsulated cells alone and with PRP at different incubation period (day 1,3 , and 5 ) in a 96-well plate. This method is based on the reduction of the yellow MTT-salt [3-(4,5-dimethylthiazol-2-yl)-2, 5-diphenyltetrazolium-bromide] to blue-purple formazan crystals by mitochondrial enzyme succinate dehydrogenase. For this purpose, $1 \times 10^{4}$ cells (encapsulated) were plated on each well with PRP (controls without PRP). Then $200 \mu \mathrm{L}$ MTT solution $(5 \mathrm{mg} / \mathrm{mL}$ ) was added in each well andthe plate was incubated at $37^{\circ} \mathrm{C}$ for $3-4$ hours. Then the supernatant was replaced with $100 \mu \mathrm{L}$ DMSO in order to dissolve formazan crystal producing purple-blue colour. Optical density measured by using microplate readerat $570 \mathrm{~nm}$.

\section{In vitro chondrogenic differentiation of encapsulated rBMMSCs}

After passage 3 , the cells, at a concentration of $2 \times 10^{6} \mathrm{cell} / \mathrm{s} / \mathrm{ml}$, encapsulated in collagen solution then cultured in the 24-well tissue culture plate. After $4 \mathrm{~h}$ incubation, differentiation groups were divided to three groups. 1. TGF- $\beta 1$ group: treated with chondrogenic differentiation media that contains DMEM/F12 supplemented with $10 \% \mathrm{ITS}+10^{-7} \mathrm{M}$ dexamethasone (Sigma), $1 \mu \mathrm{M}$ ascorbate-2-phosphate, and $10 \mathrm{ng} / \mathrm{ml}$ transforming growth factor-beta 1 (TGF- $\beta 1$, SIGMA,USA) 2. PRP- TGF- $\beta 1$ group: treated with chondrogenic differentiation media +PRP with a ratio of 1:1, and 3 . Control group without differentiation media and PRP. The cell culture maintained for 21 days with media change in every 3 days. Finally, the cells were collected for the real time PCR and were fixed for immunocytochemistry for expression of related genes to chondrocyte.

\section{Real time PCR}

Quantitative reverse transcriptase-polymerase chain reaction (qRT-PCR) was used for the mRNA expression patterns of chondrogenic specific genes in control, TGF- $\beta 1$ and PRP-TGF- $\beta 1$ groups. Total RNA was prepared by using RNeasy Plus Mini kit (Qiagen, USA, 74134) as described by the manufacturer, and complementary DNA (cDNA) synthesis from $1 \mu \mathrm{g}$ of extracted RNA was performed by Revert Aid First Strand cDNA Synthesis kit (Takara, USA, K1632). qRT-PCR reactions were carried out in the 48-well optical reaction plates on StepOneTM Real-Time PCR machine. For each PCR reaction, 30ng synthesized cDNA was used for PCR by mixing with $10 \mu$ l of Power SYBER Green master mix (2x, Applied Biosystems) primed with $0.5 \mu \mathrm{M}$ of each primer (Table 1) in a total volume of $20 \mu \mathrm{l}$ at the annealing temperature. The 
comparative Ct method ( $\triangle \Delta \mathrm{CT}$ Method) was used for relative gene expression analysis. All Ct values calculated from the normalization of target genes to GAPDH as an internal control and calibrated using calculation from the undifferentiated BM-MSCs. The relative gene expression values presented as mean of three independent experiments.

\section{Immunocytochemistry}

After 21 days' post-induction to chondrogenic differentiation, encapsulated cells were fixed with $4 \%$ paraformaldehyde (PFA; Sigma-Aldrich) for 20 minutes. Subsequently permeabilized with $0.1 \%$ Triton X100 in PBS. The cells were blocked for $30 \mathrm{~min}$ at room temperature with $5 \%$ bovine serum albumin (BSA), thenincubated with primary antibodies (diluted in $5 \%$ BSAin PBS)against aggrecan (mouse monoclonal antibody; Abcam, USA, 1:200), collagen type I (mouse monoclonal antibody; Abcam, 1:200), and collagen

type II (mouse monoclonal antibody; Abcam, $1: 200$ ) overnightat $4^{\circ} \mathrm{C}$. Secondary antibodies included Alexa fluor 488 donkey anti-mouse (1:500; Gibco) and the nuclei were counter-stained with DAPI (Sigma-Aldrich, D8417). For negative controls, only the secondary antibody was used.

\section{In vivo experiment}

The 8 weeks male wistar rats (200-220g) were randomized into four groups each of 6 rats: (i) OA group (OA induction without treatment), (ii) OA+MSC group, (iii) OA+Collagen+MSC+PRP group, and (iv) $\mathrm{OA}+\mathrm{MSC}+$ Collagen group. OA of the knee joints was induced as previously described by Tang et al (2017). In brief, under sterile conditions, the animals were anesthetized and a medial parapatellar approach was used to release the joint capsule and to perform a medial collateral ligament transection (MCLT) of the right knee joints. The wounds were closed and covered with a local antibiotic ointment. All rats were returned to their cages after the operation and were allowed to move freely, and $0.2 \mathrm{mg} / \mathrm{kg} / \mathrm{day}$ of intramuscular Meloxicam was administered for 5 days for pain relief and administrated by penicillin once a day for the first 3 days. On fourth weeks post-induction,OA was confirmedbyradiography. Then the knee joints were injected by MSC, Collagen+MSC, and Collagen+MSC+PRP. The recipient rats were injected with $4 \times 10^{6}$ collagen-encapsulated MSCs (passage 3). All rats were euthanized 6 weeks posttreatment, and knee joint samples were collected for histopathological evaluations.

\section{Statistical analysis}

Tukey's HSD test and repeated measures analysis of variance test was used for multigroup comparisons according to the GraphPad Prism software, Version6.00 (GraphPad Prism, Inc., San Diego, CA). $P<0.05$ was considered significant.

\section{Results}

\section{Isolation and characterization of rBMMSCs}


The isolated rBMMSCs from the rat femur and tibia bones exhibited typical fibroblastic morphology (Fig. 1A). At passages 3 , flow cytometry analysis showed that the cells were negative for CD31 and CD34 and positive for CD90 and CD105 (Fig. 1B). The adipogenic and osteogenic differentiation were approved by oil red $O$ and Alizarin red staining, respectively (Fig.1Ab, 1Ac).

\section{PRP analysis and classification}

Platelet rich plasma contained $1298900 \pm 248000$ platelets/ $\mu$ l compared to platelet level $(978000 \pm$ 164000 platelets/ $\mu \mathrm{l})$. White blood cell count (WBC) was $10000 \pm 2100 / \mu \mathrm{L}$ in plasma and $6800 \pm 3580 / \mu \mathrm{l}$ in PRP. Neutrophils/ $\mu \mathrm{L}$ in plasma was $395 \pm 220$ and in PRP $388 \pm 256$. This PRP is classified as P4-x-B according to the PAW classification system (26).

\section{MTT assay}

The proportion of live cells in the Collagen+Cell+PRP group was higher than those of in the Cell alone and Collagen+Cell groups (Fig. 2). This result confirmed that PRP increases the survival rate of MSCs. SEM image of the rBMMSCs seeded on the collagen is shown in Fig. 2A. This micrograph shows cell attachment and interaction with collagen at $48 \mathrm{~h}$ post- encapsulation.

\section{rBMMSCsencapsulation and chondrogenic differentiation}

The collagen scaffolds were successfully produced and micro-encapsulated with MSCs. All sacffolds were bead-like and remained in its original gel state during the 21 days of chondrogenic differentiation induction. All scaffolds were able to maintain and induce the most basic morphological feature of a chondrocyte, its round shape. The cell distribution was homogeneous, occupying all the scaffold area. Two hours post-seeding, the cells were in close contact with the scaffold, however, lacunae were observed housing the cells after one week, due to ECM production confirmed at histological sections. Some chondrocyte-like features were observed on the phase-contrast microscopy such as cells inside lacunae and cells grouped together resembling isogenous groups commonly found in hyaline cartilage.

\section{Cartilage genes expression}

At 21 day of differentiation, the expression of chondrogenic of genes collagen types I type II, and aggrecan, in various groups were analyzed with qRT-PCR. The chondrogenic genes expressions were upregulated and these levels in group of TGF- $\beta 1$ and PRP-TGF- $\beta 1$ were obviously higher than that of in the control group. Furthermore, aggrecan expression in the PRP-TGF- $\beta 1$ significantly higher than that of the TGF- $\beta$ group (Fig.3).

\section{Chondrogenic differentiation}

The effect of PRP on cartilage-specific proteins is shown in Fig.4. Collagen types I, II and aggrecan are the main structural proteins of extracellular matrix (ECM) in cartilage. In the PRP-TGF- $\beta 1$ group, the mean amount of collagen types I, II and aggrecan proteins was significantly more than that of TGF- $\beta 1$ group. In 
comparison, when the cells were treated with PRP along with TGF- $\beta 1$, they synthesized more collagen types I, II and aggrecan than that of the TGF- $\beta 1$ group (Fig.4).

\section{In vivo experiment}

Osteoarthritis was confirmed by radiographic evaluation indicating the severe loss of joint space in medial compartment using (Fig. 5,6). Histopathological features of the knee joint in various groups are shown in the Figs 7 and 8 . Histologically, the control group showed severe abrasion or superficial fibrillation characterized by microscopic cracks into the superficial, mid and deep zones of articular cartilage (AC) (Fig. 7A). In the control group, cartilage matrix loss led to delamination of superficial, mid, and deep layers and cyst formation associated with chondrocyte death, regeneration (clusters), replication, hypertrophy, and proliferation. The deformative change also caused articular bone plate micro-fracture. The organization of the trabecular bone and subchondral was disrupted. Sever subchondral sclerosis and extensively increasing bone volume and bone marrow (BM) distance from cartilage was also identified. Finally, osteonecrosis associated with the infiltration of osteoclastic-like cells associated with severe infiltration of multinucleated giant cells indicating granulomatous inflammation and area of bone resorption was detected in the control group.

In the OA+MSC group, moderate abrasion or superficial fibrillation characterized by microscopic cracks into the mid and deep zones of AC was seen (Fig. 7B). Mild fibrillation characterized by microscopic cracks into the deep zone of $\mathrm{AC}$ was detected in the $\mathrm{OA}+\mathrm{MSC}+$ Collagen group (Fig. 7C). A mild subchondral sclerosis as well as micro-fractures in the trabecular bone was seen (Fig. 7C). All histopathological features including articular bone plate micro-fracture, chondrocytes proliferation, clusters and death, and osteonecrosis without infiltration of multinucleated giant was also detected in the $\mathrm{OA}+\mathrm{MSC}$ group with the lesser severity than that of the OA+MSC+Collagen group. In the $\mathrm{OA}+\mathrm{MSC}+\mathrm{Collagen}$, the disrupted organization of the subchondral bone was improved compared to both control and OA+MSC groups. The OA+MSC group also showed the better organization of the subchondral bone than that of the control group. No microscopic cracks into all three zones of AC were seen in the $\mathrm{OA}+\mathrm{MSC}+$ Collagen+PRP group. In the most part of knee joint the cartilage was intact and the cartilage matrix swelling led to cartilage hypertrophy in this group. No evident subchondral sclerosis was detected. The trabecular bone and subchondral plate were approximately intact. The induced osteoarthritis was improved in the MSC+PRP+Collagen group compared to other treatment groups as well as the control group (Fig. 7D). High magnifications of histopathological features are shown in Fig.8.

\section{Discussion}

Knee OA (KOA) is one of the most prevalent degenerative joint diseases with persistent pain and loss of function. It was published that among elders, the risk of lower limb movement disability caused by KOA is at least $40 \%$ (27). Natural polymers such as collagen has been studied the most owing to their biocompatibility, making them suitable for cell therapy (28). Tissueengineering approaches using MSCs and scaffolds is new strategy for cartilage regeneration(29). Therapies with encapsulated MSCs have 
been new approach for knee OA. Encapsulated MSCs show prolonged survival subsequent injection that could be beneficial for long-term efficacy (30). However, theabsence of functional inducing chondrogenic differentiation is drawback. In this study, the effect of cartilage regenerative capacity of a tissueengineered composite of PRP + MSCs + collagen hydrogel was evaluatedin vitro and in vivo.

The growth factors, such as TGF- $\beta$,indicate that has important capacity as a tool for stimulating chondrocyte proliferation and repair of cartilage defects (31). PRP is obtained from peripheral blood, which has pain-relief and anti-inflammatory effects, exhibited good efficacy in knee-osteoarthritis and other musculoskeletal morbidity (32). In our study, we investigated that collagen hydrogel that contain MSCs and PRP might improve the efficiency of injured articular cartilage treatment in animal model. We theorized that PRP affects MSC proliferation and differentiation, especially chondrogenic differentiation.

Drengk et al. reported that PRP activated proliferation of mesenchymal stem cells in 3D culture(33). PRP as a source of important growth factors could efficiently facilitate differentiation and proliferation of MSCs(34). In this study, the viability and proliferation of MSCs was evaluated by MTT assay. The proliferation rate of MSCs-collagen-PRP group significantly higher that other groups. PRP contains a natural cocktail of growth factors such as TGF- $\beta$ demonstratedupregulatedcartilage gene expression and differentiation (33). The capacity of chondrogenic effects of PRP are attributed to growth factors such a TGF- $\beta$, vascular endothelial growth factor, basic fibroblast growth factor produced in the alpha granules of platelets (35). In this study, Immune-staining of differentiated cells in TGF- $\beta$ - PRP group shown that, aggrecan, collagen typel and collagen typell protein expression are higher than TGF- $\beta$-PRP group and confirmed that enhancive effect of TGF- $\beta$ transcription factor in chondrogenic differentiation.Next, we investigated the gene expression of chondrogenic pathway. Zhu et al. reported that TGF- $\beta$ is reported to initiate the chondrogenic differentiation process by induction of collagen and aggrecan genes (36). Collagen typel, collagen typell and aggrecan are the main genes of cartilage extracellular matrix and play an essential role in maintenance of cartilage $(28,37)$.The gene expression of aggrecan in PRP-TGF- $\beta 1$ group was higher than that of PRP and control groups. In this study, RNA levels of cartilage-specific markers, including collagen typel, collagen typell and aggrecan were similarly higher in the PRP-TGF- $\beta 1$ and TGF- $\beta 1$ group than control group. Liou et al. found that MSCs encapsulated in a PRP-containing hydrogel, resulting in optimal MSC chondrogenic differentiation (38). Intra-articular injection of MSCs has limitations being considered to cell death after injection and leakage from the injection site. Encapsulation of MSCs by biomaterial has therefore been developed to overcome these drawbacks and to deliver therapeutic factors in intra-articular knee(39). Rogan et al. reported thatMSCs encapsulation obviously resulted in more cartilage formation in KOA models. Luca et al, founded that encapsulated cells show long-standing survival following injection that could be useful for long-term efficacy of MSCs in cell-based therapy of OA. In currentstudy, histological assessment of articular cartilage was conducted 6week post injection. Cartilage repair exhibited better healing result in MSC-PRP-Collagen group compared to other groups.Although the chondrogenic differentiation effect of PRP was poorer than TGF- $\beta$. The result is consistent with the research of Chellini et al. (40), and the anti-fibrotic potential of PRP underlies the formation of high-quality cartilage. 


\section{Conclusions}

Our study revealed that MSCs encapsulated in a PRP-containing collagen hydrogelcan be used as a potent chondrogenic inducer substitute on cartilage tissueengineering. In vivo experiment demonstrated that these composite have a beneficial effect on OA treatment via the stimulation of ECM synthesis and chondrocyte proliferation and provides a new insight into cartilage tissue engineering strategies.

\section{Abbreviations}

ACl: Autologous Chondrocyte Implantation, BM: Bone Marrow, BMMSCs: Bone marrow derived Mesenchymal Stem Cells, cDNA: Complementary DNA, DMEM: Dulbecco's Modified Eagle's Medium, ECM: Extracellular Matrix, FBS: Fetal Bovine Serum, FACS: Flow Cytometry FN: Fibronectin, HA: Hyaluronic Acids, MCLT: Medial Collateral Ligament Transection, KOA: Knee Osteoarthritis, MSCs: Multipotent mesenchymal stem cells, NSAIDs: Non-Steroidal Anti-Inflammatory Drugs, OA: Osteoarthritis, PBS: Phosphate Buffered Saline, PCM: Pericellular Matrix, PPP: Platelet-Poor-Plasma, PRP: Platelet-Rich Plasma, qRT-PCR: Quantitative Reverse Transcriptase-Polymerase Chain Reaction, rBMMSCs: Rat Bone marrow derived Mesenchymal Stem Cells

\section{Declarations}

\section{Ethics approval and consent to participate:}

Animal experiments were approved by the ethics committee of the Tehran university of medical sciences (ethical code: IR.TUMS. VCR.1398.034) and were carried out in accordance with the university's guidelines.

ETHICAL STATEMENT:

\section{Ethics approval and consent to participate:}

Animal experiments were approved by the ethics committee of the Tehran university of medical sciences (ethical code: IR.TUMS. VCR.1398.034).

\section{Acknowledgment and funding:}

The authors thanks Tehran University of Medical Sciences to support of this project with grant number 96-02-103-39604.

\section{Conflicts of interest/Competing interests:}

The authors have no conflict of interest

\section{Availability of data and material}


All data are available based on request via contacting with corresponding authors by email.

\section{Consent to participate}

NA

\section{Consent for publication}

NA

\section{Authors' contributions:}

\section{Authors' Contributions}

Md Shahidul Islamcontributed to all experimental work and drafted the manuscript. Mamun Al Mahtabrevised the manuscript and contributed to the interpretation of data.Sadegh Shirianparticipated to the establishing animal model and surgical study.Hamid Reza Aghayan and Babak Arjmandcontributed to the study design and stem cell isolation.Vajiheh Taghdiri Nooshabadiparticipated in evaluation and characterization of PRP.Amin Bigham Sadeghparticipated in histological and radiographical study. Amir Allahverdicontributed in molecular analysis. Faezeh Esmaeili Ranjbarcontributed in stem cell characterization. Jafar Ai and Somayeh Ebrahimi-Barough were responsible for the overall supervision, contributed to design research, data interpretation and manuscript revision.

\section{References}

1. Burchard R, Huflage H, Soost C, Richter O, Bouillon B, Graw JA. Efficiency of platelet-rich plasma therapy in knee osteoarthritis does not depend on level of cartilage damage. Journal of orthopaedic surgery and research. 2019;14(1):153.

2. Vinatier $C$, Guicheux J. Cartilage tissue engineering: From biomaterials and stem cells to osteoarthritis treatments. Annals of physical and rehabilitation medicine. 2016;59(3):139-44.

3. Grässel S, Lorenz J. Tissue-engineering strategies to repair chondral and osteochondral tissue in osteoarthritis: use of mesenchymal stem cells. Current rheumatology reports. 2014;16(10):452.

4. Wang T, Yang F. A comparative study of chondroitin sulfate and heparan sulfate for directing threedimensional chondrogenesis of mesenchymal stem cells. Stem cell research \& therapy. 2017;8(1):284.

5. Gikas P, Bayliss L, Bentley G, Briggs T. An overview of autologous chondrocyte implantation. The Journal of bone and joint surgery British volume. 2009;91(8):997-1006.

6. Oryan A, Alidadi S, Moshiri A, Maffulli N. Bone regenerative medicine: classic options, novel strategies, and future directions. Journal of orthopaedic surgery and research. 2014;9(1):1-27.

7. Bornes TD, Adesida AB, Jomha NM. Mesenchymal stem cells in the treatment of traumatic articular cartilage defects: a comprehensive review. Arthritis research \& therapy. 2014;16(5):432. 
8. Gattegno-Ho D, Argyle S-A, Argyle DJ. Stem cells and veterinary medicine: tools to understand diseases and enable tissue regeneration and drug discovery. The Veterinary Journal. 2012;191(1):1927.

9. Nooshabadi V. Endometrial Mesenchymal Stem Cell-Derived Exosome Promote Endothelial Cell Angiogenesis in a Dose Dependent Manner: A New Perspective on Regenerative Medicine and CellFree Therapy. Archives of Neuroscience. 2019(In Press).

10. Xia H, Liang C, Luo P, Huang J, He J, Wang Z, et al. Pericellular collagen I coating for enhanced homing and chondrogenic differentiation of mesenchymal stem cells in direct intra-articular injection. Stem cell research \& therapy. 2018;9(1):174.

11. Mak J, Jablonski C, Leonard C, Dunn JF, Raharjo E, Matyas J, et al. Intra-articular injection of synovial mesenchymal stem cells improves cartilage repair in a mouse injury model. Scientific reports. 2016;6:23076.

12. Jo CH, Lee YG, Shin WH, Kim H, Chai JW, Jeong EC, et al. Intra-articular injection of mesenchymal stem cells for the treatment of osteoarthritis of the knee: A proof-of-concept clinical trial. Stem cells. 2014;32(5):1254-66.

13. Leijs MJ, van Buul GM, Verhaar JA, Hoogduijn MJ, Bos PK, van Osch GJ. Pre-treatment of human mesenchymal stem cells with inflammatory factors or hypoxia does not influence migration to osteoarthritic cartilage and synovium. The American journal of sports medicine. 2017;45(5):1151-61.

14. Moshaverinia A, Xu X, Chen C, Akiyama K, Snead ML, Shi S. Dental mesenchymal stem cells encapsulated in an alginate hydrogel co-delivery microencapsulation system for cartilage regeneration. Acta biomaterialia. 2013;9(12):9343-50.

15. Bian L, Zhai DY, Tous E, Rai R, Mauck RL, Burdick JA. Enhanced MSC chondrogenesis following delivery of TGF- $\beta 3$ from alginate microspheres within hyaluronic acid hydrogels in vitro and in vivo. Biomaterials. 2011;32(27):6425-34.

16. Mehranfar S. The use of stromal vascular fraction (SVF), platelet-rich plasma (PRP) and stem cells in the treatment of osteoarthritis: an overview of clinical trials. Artificial cells, nanomedicine, and biotechnology. 2019; 47(1): 882-890.

17. ParmarPA, Chow LW, St-Pierre JP, Horejs CM, Peng YY, Werkmeister JA, Ramshaw JA, Stevens MM. Collagen-mimetic peptidemodifiable hydrogels for articular cartilage regeneration, Biomaterials. 2015; 54: 213-225

18. Raghothaman D, Leong MF, Lim TC, Toh JK, Wan AC, Yang Z, et al. Engineering cell matrix interactions in assembled polyelectrolyte fiber hydrogels for mesenchymal stem cell chondrogenesis. Biomaterials. 2014;35(9):2607-16.

19. Singh P, Schwarzbauer JE. Fibronectin and stem cell differentiation-lessons from chondrogenesis. Journal of cell science. 2012;125(16):3703-12.

20. Erat MC, Sladek B, Campbell ID, Vakonakis I. Structural analysis of collagen type I interactions with human fibronectin reveals a cooperative binding mode. Journal of Biological Chemistry. 2013;288(24):17441-50. 
21. Cohen M, Joester D, Geiger B, Addadi L. Spatial and temporal sequence of events in cell adhesion: from molecular recognition to focal adhesion assembly. Chembiochem. 2004;5(10):1393-9.

22. Yun S, Ku S-K, Kwon Y-S. Adipose-derived mesenchymal stem cells and platelet-rich plasma synergistically ameliorate the surgical-induced osteoarthritis in Beagle dogs. Journal of orthopaedic surgery and research. 2016;11(1):9.

23. Sampson S, Reed M, Silvers H, Meng M, Mandelbaum B. Injection of platelet-rich plasma in patients with primary and secondary knee osteoarthritis: a pilot study. American Journal of Physical Medicine \& Rehabilitation. 2010;89(12):961-9.

24. Van Pham P, Bui KH-T, Ngo DQ, Vu NB, Truong NH, Phan NL-C, et al. Activated platelet-rich plasma improves adipose-derived stem cell transplantation efficiency in injured articular cartilage. Stem cell research \& therapy. 2013;4(4):91.

25. Rajan N, Habermehl J, Coté M-F, Doillon CJ, Mantovani D. Preparation of ready-to-use, storable and reconstituted type I collagen from rat tail tendon for tissue engineering applications. Nature Protocols. 2006;1(6):2753-8.

26. DeLong JM, Russell RP, Mazzocca AD. Platelet-rich plasma: the PAW classification system. Arthroscopy: the journal of arthroscopic \& related surgery: official publication of the Arthroscopy Association of North America and the International Arthroscopy Association. 2012;28(7):998-1009. pmid:22738751.

27. Johnson VL, Hunter DJ. The epidemiology of osteoarthritis. Best practice \& research Clinical rheumatology. 2014;28(1):5-15.

28. Pak J, Lee JH, Pak N, Pak Y, Park KS, Jeon JH, et al. Cartilage Regeneration in Humans with Adipose Tissue-Derived Stem Cells and Adipose Stromal Vascular Fraction Cells: Updated Status. Int J Mol Sci. 2018;19(7).

29. Mardones R, Jofré CM, Minguell JJ. Cell therapy and tissue engineering approaches for cartilage repair and/or regeneration. International journal of stem cells. 2015;8(1):48.

30. Jiang $X$, Huang $X$, Jiang $T$, Zheng $L$, Zhao J, Zhang $X$. The role of Sox 9 in collagen hydrogel-mediated chondrogenic differentiation of adult mesenchymal stem cells (MSCs). Biomaterials science. 2018;6(6):1556-68.

31. Bakker A, Van De Loo F, Van Beuningen H, Sime P, Van Lent P, Van Der Kraan P, et al. Overexpression of active TGF-beta-1 in the murine knee joint: evidence for synovial-layer-dependent chondroosteophyte formation. Osteoarthritis and cartilage. 2001;9(2):128-36.

32. Andia I, Abate M. Platelet-rich plasma: underlying biology and clinical correlates. Regenerative medicine. 2013;8(5):645-58.

33. Drengk A, Zapf A, Stürmer EK, Stürmer KM, Frosch K-H. Influence of platelet-rich plasma on chondrogenic differentiation and proliferation of chondrocytes and mesenchymal stem cells. Cells Tissues Organs. 2009;189(5):317-26.

34. Atashi F, Jaconi ME, Pittet-Cuénod B, Modarressi A. Autologous platelet-rich plasma: a biological supplement to enhance adipose-derived mesenchymal stem cell expansion. Tissue Engineering Part 
C: Methods. 2015;21(3):253-62.

35. Boswell SG, Cole BJ, Sundman EA, Karas V, Fortier LA. Platelet-rich plasma: a milieu of bioactive factors. Arthroscopy: The journal of arthroscopic \& related surgery. 2012;28(3):429-39.

36. Zhu Y, Tao H, Jin C, Liu Y, Lu X, Hu X, et al. Transforming growth factor- $\beta 1$ induces type II collagen and aggrecan expression via activation of extracellular signal-regulated kinase 1/2 and Smad2/3 signaling pathways. Molecular Medicine Reports. 2015;12(4):5573-9.

37. Sekiya I, Tsuji K, Koopman P, Watanabe H, Yamada Y, Shinomiya K, et al. SOX9 enhances aggrecan gene promoter/enhancer activity and is up-regulated by retinoic acid in a cartilage-derived cell line, TC6. Journal of Biological Chemistry. 2000;275(15):10738-44.

38. Liou J-J, Rothrauff BB, Alexander PG, Tuan RS. Effect of platelet-rich plasma on chondrogenic differentiation of adipose-and bone marrow-derived mesenchymal stem cells. Tissue Engineering Part A. 2018;24(19-20):1432-43.

39. Hached F, Vinatier C, Le Visage C, Gondé H, Guicheux J, Grimandi G, et al. Biomaterial-assisted cell therapy in osteoarthritis: From mesenchymal stem cells to cell encapsulation. Best Practice \& Research Clinical Rheumatology. 2017;31(5):730-45.

40. Chellini F, Tani A, Vallone L, Nosi D, Pavan P, Bambi F, et al. Platelet-rich plasma prevents in vitro transforming growth factor- $\beta 1$-induced fibroblast to myofibroblast transition: involvement of vascular endothelial growth factor (VEGF)-A/VEGF receptor-1-mediated signaling. Cells. $2018 ; 7(9): 142$.

\section{Tables}

Table 1. Primers sequences for real time-PCR

$\begin{array}{lll}\text { primer forward } & \text { Accessation }\end{array}$ number

$\begin{array}{llll}\text { Aggrecan } & \text { TCCACATCAGAAGAGCCATAC } & \text { AGTCAAGGTCGCCAGAGG } & \text { NM_022190.1 } \\ \begin{array}{l}\text { Collagen } \\ \text { I }\end{array} & \text { GCTGTGGAAGTGGATGAAGA } & \text { TGAGGAACTGTGGAGAGACG } & \text { NM_053304 } \\ \begin{array}{l}\text { Collagen } \\ \text { II }\end{array} & \text { ACCTGGTACCCCTGGAAATC } & \text { CACCAGGATTGCCTTGAAAT } & \text { NM_012929.1 } \\ \text { GAPDH } & \text { TGAGGACCAGGTTGTCTCCT } & \text { ATGTAGGCCATGAGGTCCAC } & \text { NM_017008.4 }\end{array}$

\section{Figures}



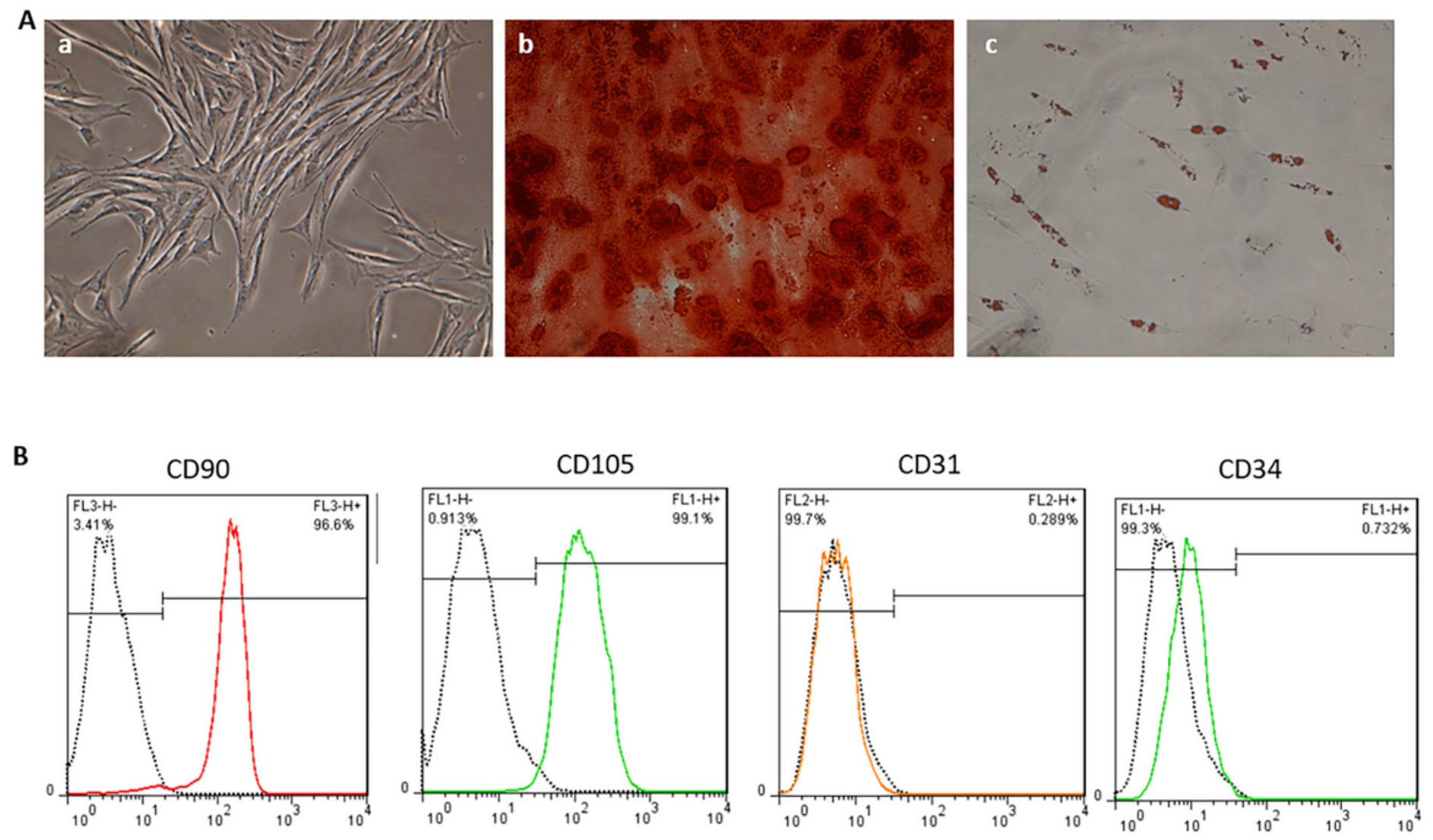

\section{Figure 1}

Bone Marrow derived Mesenchymal stem isolation and characterization. (A) Cell isolation and differentiation. (a) Isolated MSCs showed spindle and fibroblast-like shapes. (b) Osteogenic and adipogenic differentiation were visualized by Alizarin Red and Oil Red $O$ staining respectively. (B) CD marker characterization by flow cytometry (FACS). In this representative data set, the cells were positive for CD90 and CD105, negative for CD31 and CD34. 
A
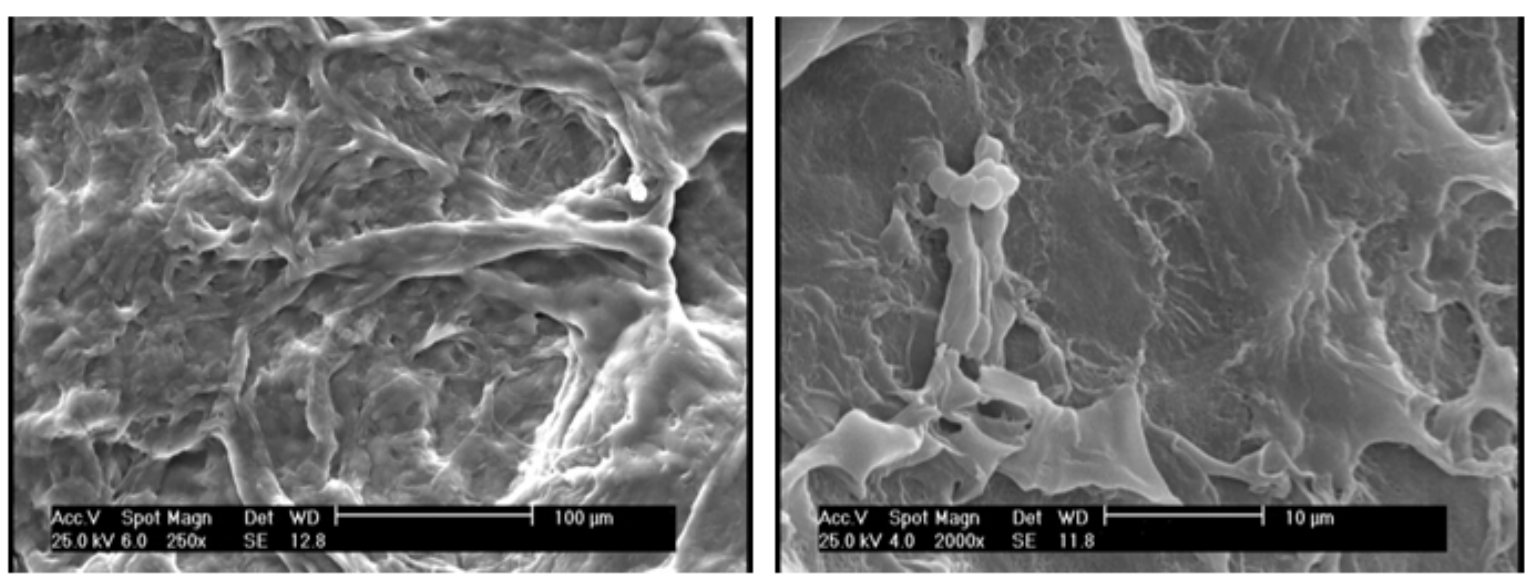

B

1.4

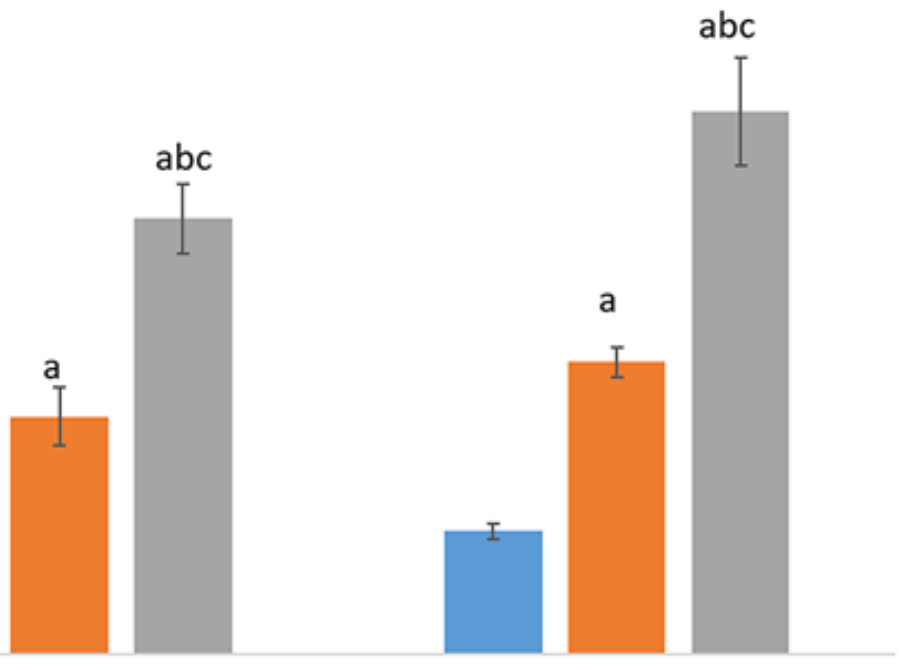

DAY1

DAY3

DAY5

n cell $\square$ collagen+cell $\square$ collagen+cell+PRP

\section{Figure 2}

Cell attachment and viability in collagen scaffold. A) SEM micrograph from encapsulated cells in collagen after $48 \mathrm{~h}$. B) Cells viability of BMMSCs with basal culture medium as a control group, collagen encapsulation with basal culture medium, collagen encapsulation with basal culture medium

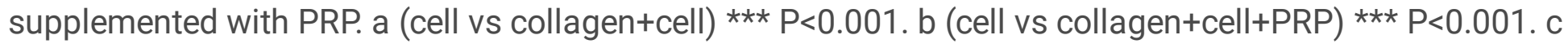
(collagen+cell vs collagen+cell+PRP) ${ }^{\star \star \star} \mathrm{P}<0.001$ 
6

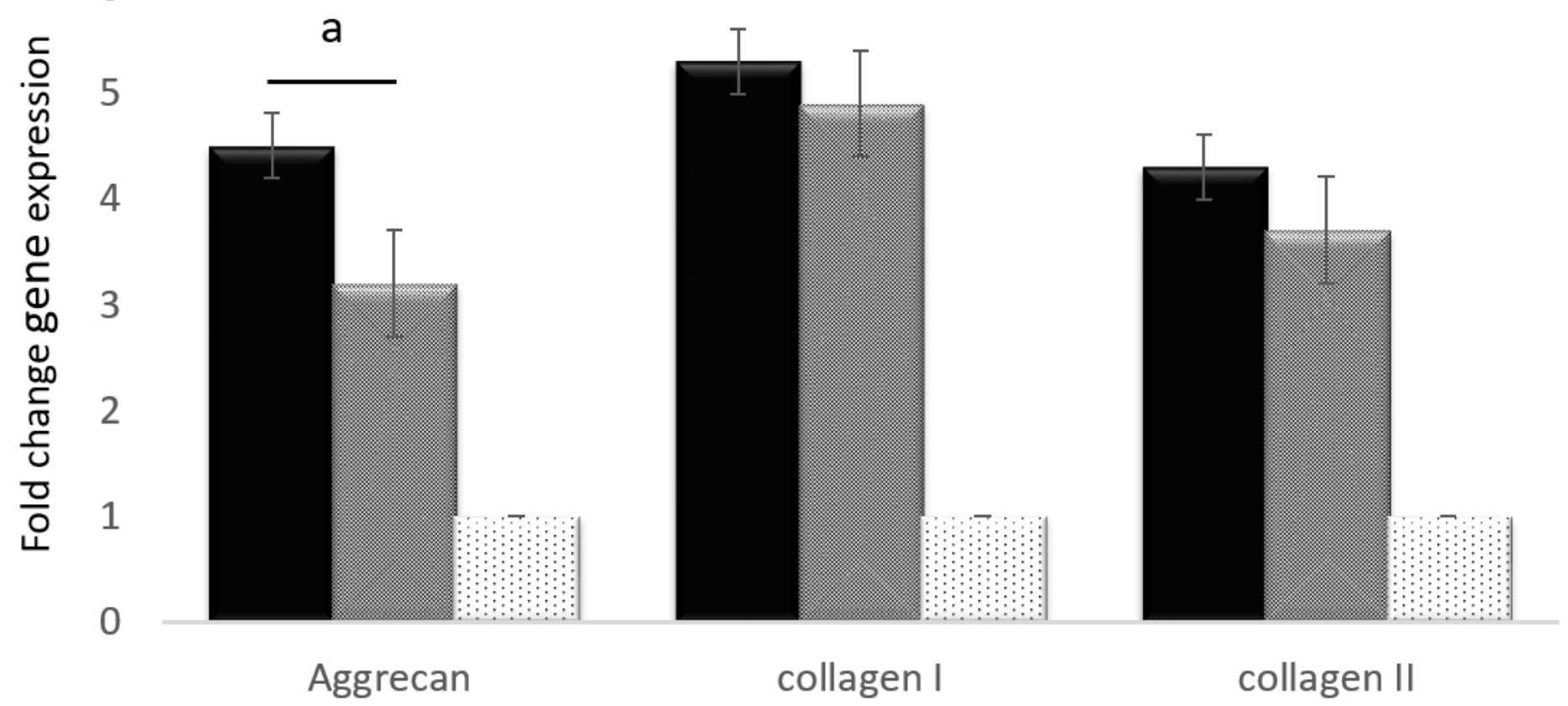

口TGF-b a PRP il control

\section{Figure 3}

Expression of cartilage-specific genes. Relative expression of aggrecan, collagen type I, collagen type II in control, TGF- $\beta 1$ and PRP- TGF- $\beta 1$ groups during chondrogenic differentiation. The expressions of collagen type I, collagen type II and aggrecan genes were significantly upregulated in TGF- $\beta$ compared to that in control group. Results are presented as mean \pm SD. Significant levels are a $p<.001$. 


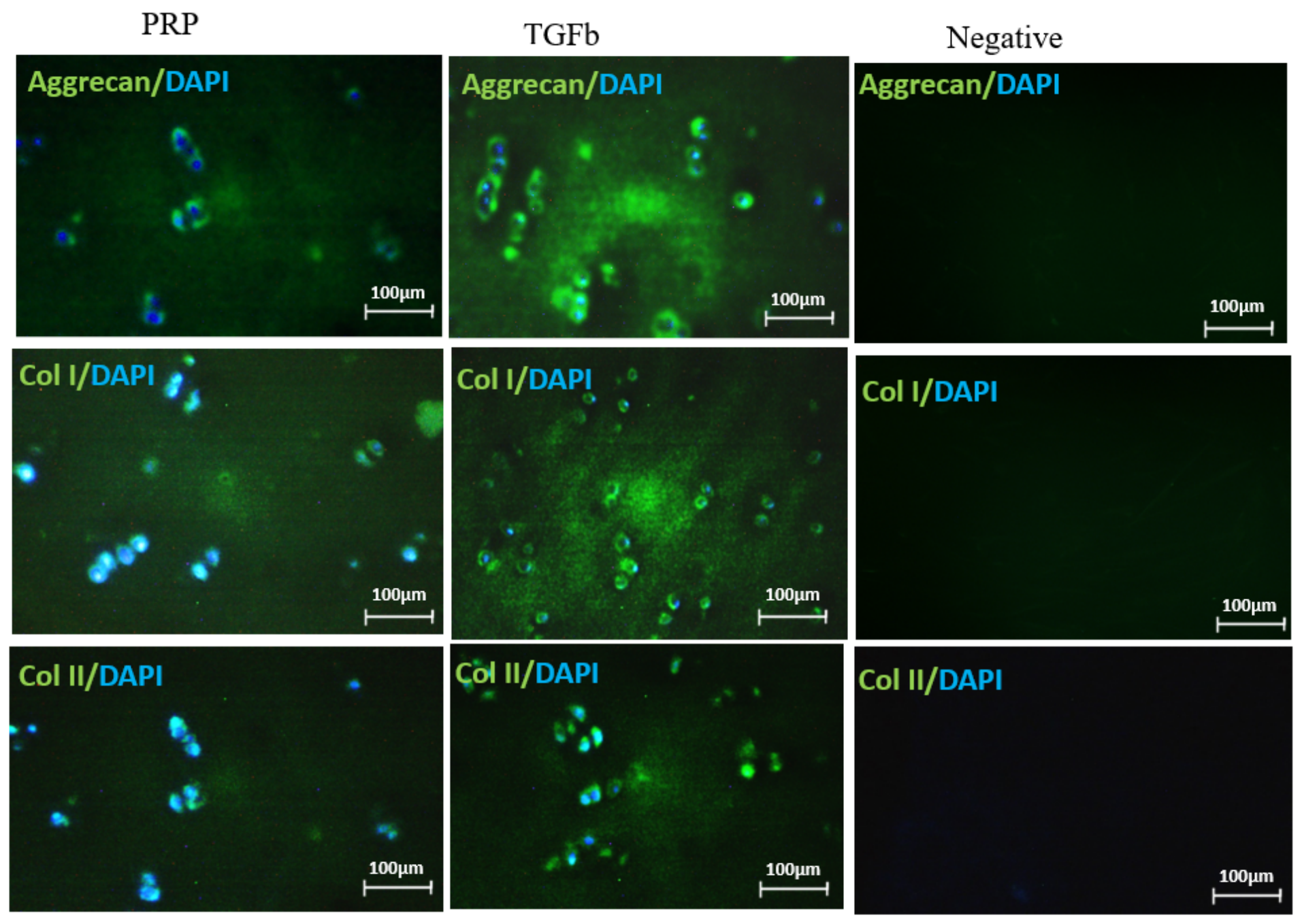

Figure 4

The effect of PRP and TFG- $\beta 1$ on the biosynthesis of the extracellular matrix, as detected by immunofluorescence. (A) Cells were stained for Aggrecan (green), collagen type I (green), collagen type II (green), and nuclei were stained with DAPI (blue) after 21 days in culture. 


\section{Figure 5}

The X-ray assay shows the loss of joint space in medial compartment indicating Osteoarthritis (black arrow). 

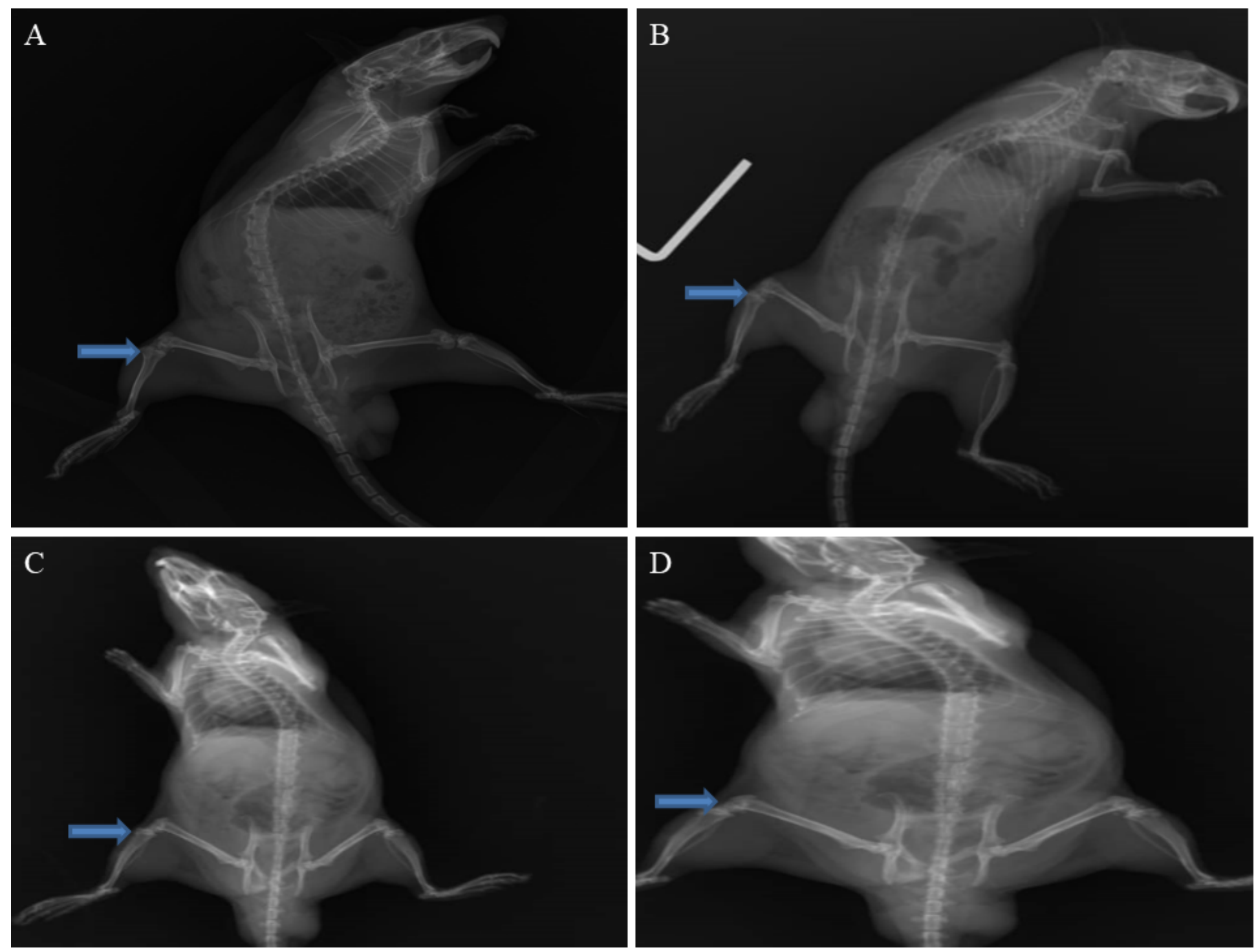

\section{Figure 6}

Radiographical images the severe osteoarthritis indicated by loss of joint space in the control group ( $A$, arrow), moderate osteoarthritis in the MSC group (B, arrow), mild osteoarthritis in the MSC+Collagen group (C, arrow), and without osteoarthritis in the MSC+Collagen+PRP group (D, arrow) 

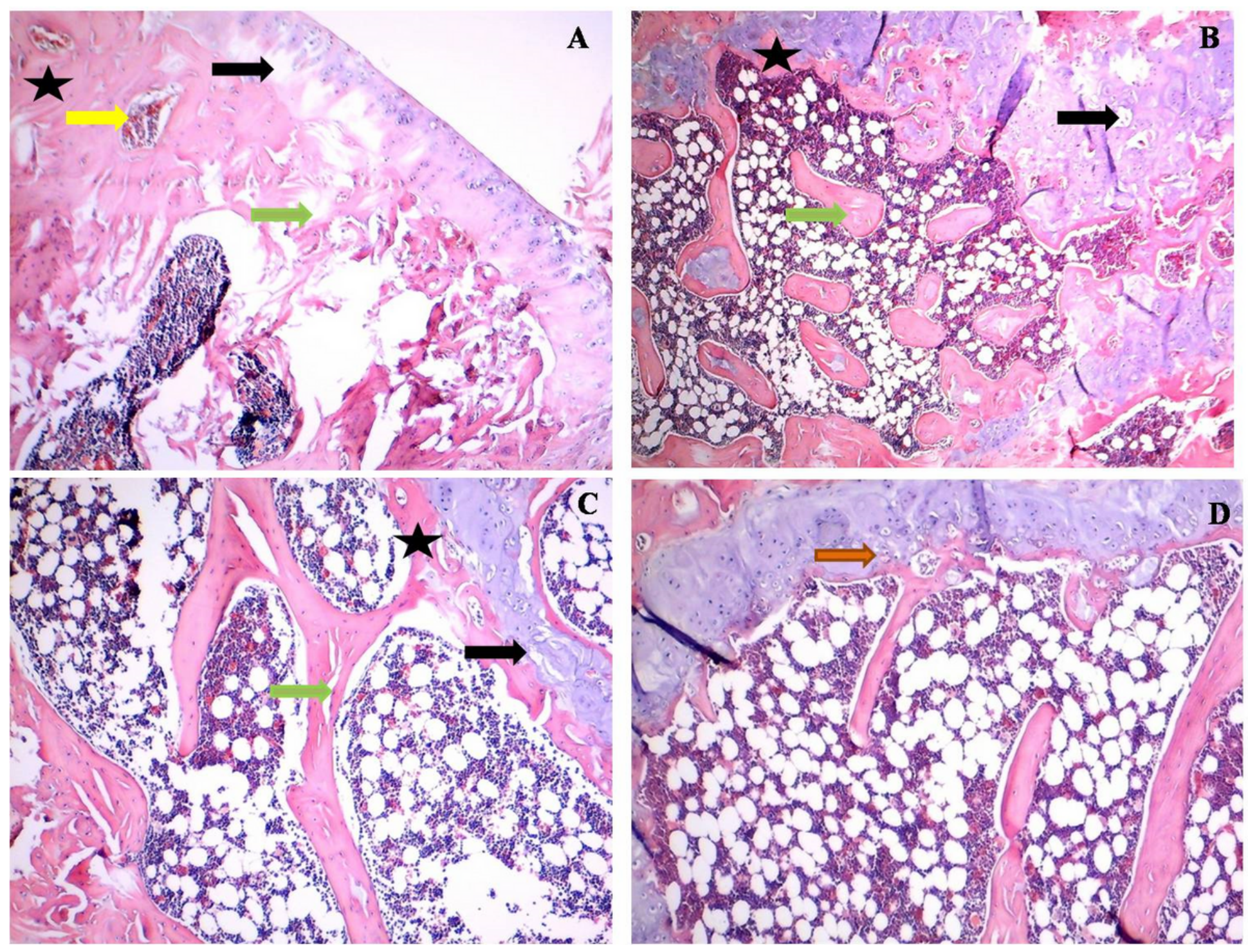

Figure 7

Histopathological findings of the knee joint in various groups, A: Cartilage matrix loss resulted in delamination of superficial, mid, and deep layers and cyst formation (black arrow) and vascular invasion in subchondral bone (yellow arrow). The organization of the subchondral and trabecular bone was disrupted (green arrow). Severe subchondral sclerosis and extensively increased in bone volume (star) and bone marrow (BM) distance from cartilage was seen. Osteonecrosis associated with the area of trabecular bone fracture (green arrow) was identified in the control or osteoarthritis (OA) group. B: in the OA+MSC group, mild cartilage matrix loss resulted in delamination of mid and deep layers and cyst formation (black arrow). The organization of the subchondral and trabecular bone was disrupted (green arrow). A distinct subchondral sclerosis (star) was seen. Osteonecrosis associated with the area of trabecular bone fracture (green arrow) was identified. C: mild cartilage matrix loss resulted in delamination of mid and deep layers and cyst formation (black arrow). The organization of the subchondral and trabecular bone was disrupted (green arrow). A mild subchondral sclerosis (star) was seen. Osteonecrosis associated with the area of trabecular bone fracture (green arrow) was identified in the OA+MSC+Collagen groups. D: There were no microscopic cracks into all three zones of articular 
cartilage in the OA+MSC+Collagen+PRP group. The cartilage was intact in the most part of knee joint and cartilage matrix swelling leads to cartilage hypertrophy in this group (brown arrow). There was no evident subchondral sclerosis. The subchondral plate and trabecular bone was approximately intact.
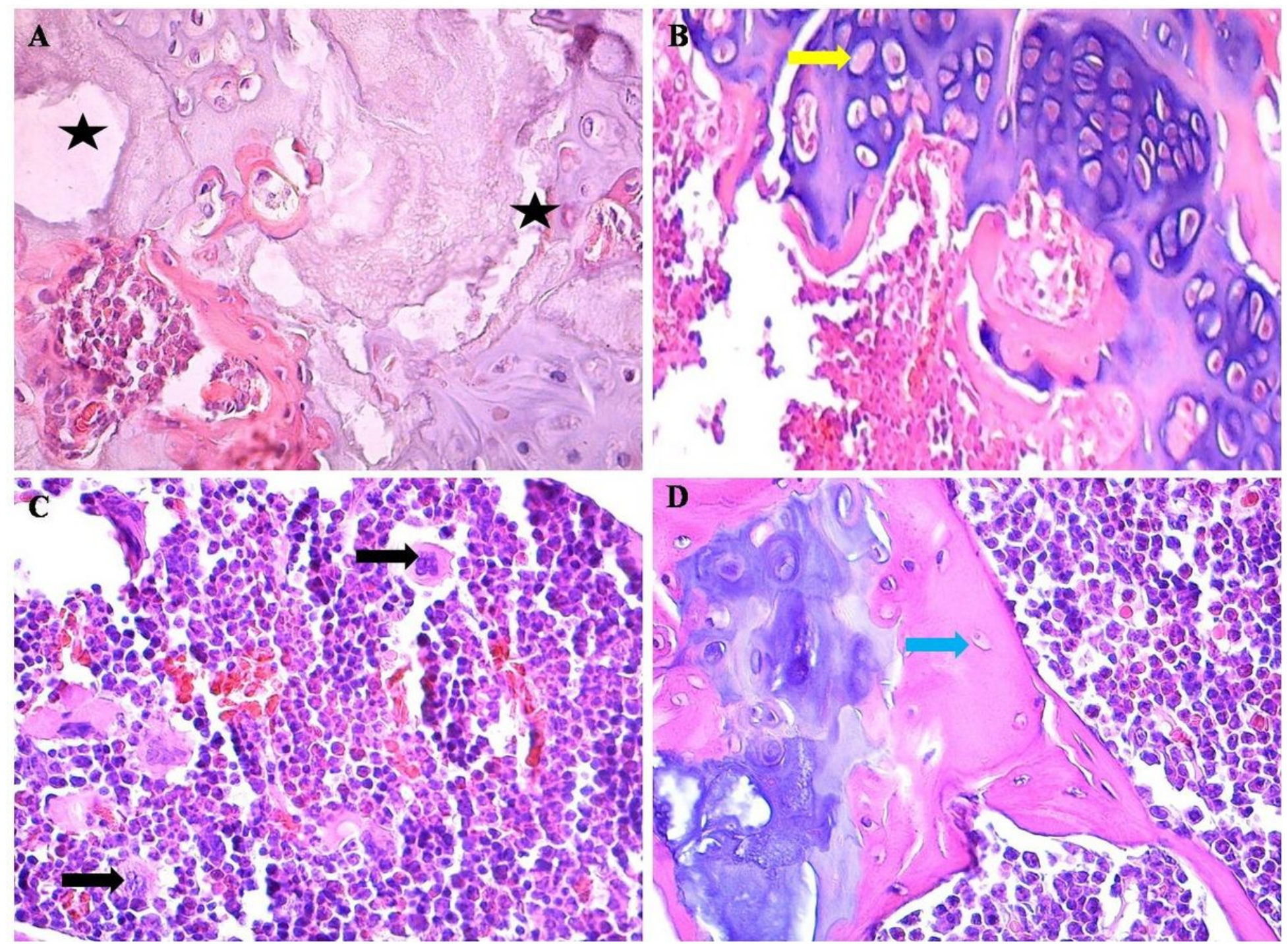

Figure 8

High magnification of histological findings: A, cartilage matrix loss leading to cyst formation (stars); B; chondrocyte death (yellow arrow), regeneration (clusters), and hypertrophy; C: severe infiltration of multinucleated giant cells (black arrow) indicating granulomatous inflammation; D: osteonecrosis

\section{Supplementary Files}

This is a list of supplementary files associated with this preprint. Click to download.

- Graphicalabstract.png 